\title{
Ultrasound Methods for Lower Rectal Cancer Staging
}

\author{
Ludmila Tankova, and Stanislav Churchev* \\ Clinical Center of Gastroenterology, University Hospital 'Queen Joanna', 1527 Sofia, 8 Belo more str., Bulgaria
}

Received: September 15, 2014; Accepted: December 01, 2014; Published: December 10, 2014

*Corresponding author: Stanislav Churchev MD, Clinical Center of Gastroenterology, University Hospital 'Queen Joanna', 1527 Sofia, 8 Belo more str., Bulgaria, Tel: +359-886-738-307; E-mail: churchev@gmail.com

\begin{abstract}
Accurate staging for low rectal cancer is essential for selecting patients who can undergo sphincter-preserving surgery and identifying those who could benefit from neoadjuvant therapy.

In this article we review the literature and report our experience in preoperative locoregional staging of low rectal cancer with ultrasonographic methods. Nearly 30 years after its introduction into clinical practice Endorectal Ultrasound (ERUS) is established as a main diagnostic modality for locoregional staging of colorectal cancer. The accuracy of the method for assessing the tumor invasion (T-stage) varies between $80 \%$ to $95 \%$. Endorectal ultrasound is the preferred method for rectal cancer staging in early T1 and T2 lesions. This method is less precise in evaluating lymph nodes ( $\mathrm{N}$-stage). A median accuracy of $75 \%$ is reported. With endoultrasound - guided fine needle aspiration biopsy the specificity and sensitivity of ERUS for confirming the malignant character of lymph nodes and local recurrence are improved. Three dimensional ERUS has some benefits in comparison to its 2D counterpart. The method allows better estimation of the volume of the tumor and the relation to nearby anatomical structures. In low rectal and anal carcinoma, ERUS and transperineal ultrasound can be used to evaluate the intactness of anal sphincter apparatus. Transvaginal ultrasound can be done as an addition to every ERUS examination in women with rectal cancer. This approach is very useful in cases of rectal stenotic tumors.
\end{abstract}

Keywords: Rectal cancer; Endorectal ultrasound; Transvaginal ultrasound; Transperineal ultrasound

\section{Introduction}

Rectal cancer comprises approximately one third of all colorectal carcinomas, and of these approximately a third arise < $6 \mathrm{~cm}$ from the anal verge. Accurate staging for low rectal cancer is essential for selecting patients who can undergo sphincterpreserving surgery and identifying those who could benefit from neoadjuvant therapy.

The Endorectal Ultrasound (ERUS) used for examination of the lower part of the gastrointestinal tract is also referred as transrectal ultrasound, endoluminal ultrasound, Endoscopic Ultrasonography (EUS). Endorectal ultrasonography was introduced in clinical practice for rectal cancer staging in 1985 [1].

In most of the instances rigid probes/linear, convex, multiplanar, radial/or oblique-viewing radial-array echoendoscopes are used. More rarely a miniprobe introduced through standard colonoscopies especially in stenotic lesions can be used.
The rigid linear probe may have limited insertion, but they are more practical and less expensive when compared with flexible devices. Correlation between the evaluation with radial echoendoscope and rigid linear probe indicates equivalence in the diagnostic value of both flexible and rigid devices [2].

For ERUS the patient is prepared with an enema $2 \mathrm{~h}$ before the study. ERUS is performed in the left lateral decubitus position and always starts with digital examination. Usually no sedation or general anesthesia is needed and the procedure is well tolerated by the patients. After a digital rectal examination is performed the probe covered with a balloon is inserted into the rectum. For better acoustic contact the water filled balloon (usually between 30 and $60 \mathrm{ml}$ water) is used. Some authors describe a modification of the method in which instead of the standard water-balloon filling technique, a sterile contact gel (100-150 $\mathrm{ml}$ ) was injected into the rectum directly [3]. Our practice is to introduce approximately $50 \mathrm{ml}$ of contact gel inside the balloon and a small amount of gel around it. The gel helps the probe to pass over the rectal tumor, minimizes distortion of the lesion, and improves visualization of the rectal wall. This method prevents the compression of the tumor by the balloon, which can create an image of pseudo-infiltration that can cause an overestimation of the tumor stage.

\section{Assessment of Primary Tumor by Endorectal Ultrasound}

By endorectal ultrasonography the rectal tumors can be evaluated for their size, stage, echostructure and vascularization.

Cancers in the parts of the colon beyond the rectum usually are not staged with ultrasound methods, because the primary treatment-surgical resection gives the means for definite pathohistological staging and grading. The forward-viewing radial-array echoendoscopes gives the ability to reach and evaluate these lesions. The accuracy of the collected data and its significance for clinical practice in the cases of colon tumors has to be verified by larger systematic studies [4].

The endosonographic image of rectal carcinoma is one of a hypoechoic, heterogenic lesion and the degree to which it disrupts the rectal wall layers determines the local tumor T- stage.

The transversal diameter/size of the tumor, the distance from the anal sphincters and its involvement (infiltration by the 
process) are examined. The ultrasound image of the rectal wall consists of five layers with alternating echogenic characteristics three hyperechoic and two hypoechoic layers, which correspond to the anatomic layers [5].

During endorectal ultrasonography, the internal anal sphincter appears as a well-defined ring with homogeneous hypoechogenicity, whereas the external anal sphincter usually appears hyperechoic with a heterogeneous appearance and lies immediately outside the internal sphincter. Also the loop of the puborectal muscle is well visible and indicates the proximal part of the anal canal [6]. In case of low rectal cancer involving the anal canal endorectal ultrasound can define the anal sphincter apparatus infiltration to facilitate the surgeon's decision about the operation.

\section{Evaluation of T-stage}

The accuracy of ERUS in identifying the T-stage of rectal cancer varies between $80 \%$ and 95\% [7,8] (Figures 1-Figure 3). A meta-analysis of all publications in the period 1985-2003 with 4118 cases reports median accuracy of the method of 85\% [9]. The analysis shows that the worst results are observed in later studies and that the accuracy is inversely proportional to the volume of the study. Similar results with declining accuracy in "newer" studies is also reported by other authors which shows the need for proper training, standardization of the technique of examination and the criteria for interpretation of the acquired data $[10,11]$.

The main problem and source of inaccuracy in rectal cancer staging is overemphasis of tumor invasion. Most often T2 carcinomas are diagnosed as T3 because of spontaneous (desmoplastic reaction) or iatrogenic (postbiopsic) inflammation, imitating invasion $[8,10,12]$. Also, there are difficulties in distinguishing between tumors with deep infiltration into the muscularis propria and those with minimal invasion into the perirectal fat tissue.

Sources of inaccuracy in determining the T-stage of rectal cancer are:

- Overestimation of the stage because of reactive changes: reactive inflammation, postbiopsic inflammation, desmoplastic reaction [12].

- Oblique or tangential angle of scanning: The angle of scanning affects the amplitude of the ultrasound waves which do not always pass perpendicular through the tumor, which leads to unclear visualization of the layers of the rectal wall. For instance, localization of the process over the Houston valves or near the anal canal makes the exact positioning of the ultrasound probe more difficult $[10,11]$

- Inadequate bowel preparation of the patient with retained air, stools, or mucus can create "reverberation defects" that obscure deep tumor margins and may cause overstaging. Air pockets between the probe and the tumor from air bubbles trapped in the balloon or

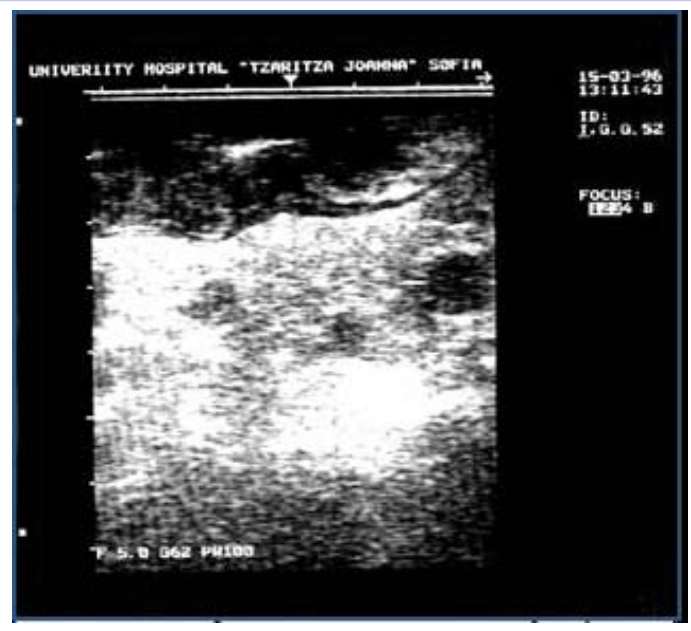

Figure 1: Endosonographic image of the rectal cancer - T2N1 stage. Disruption of the third (hypoechoic) layer.

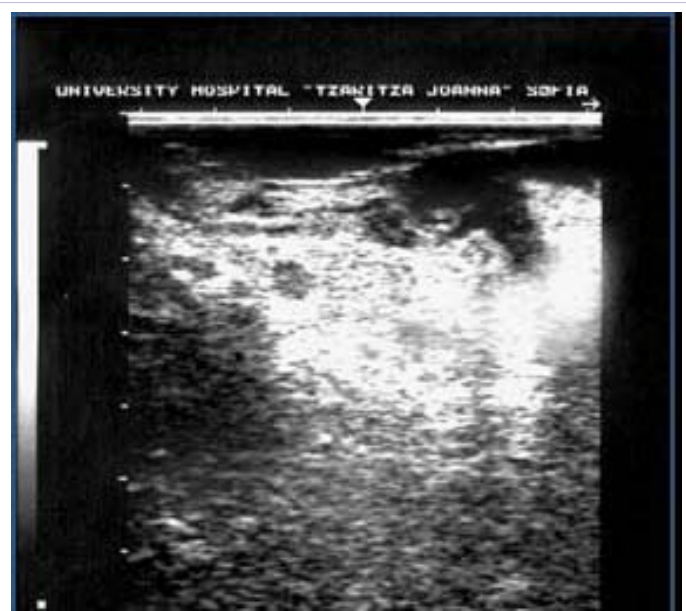

Figure 2: Endosonographic image of the rectal cancer - T3N1 stage. Extension into the perirectal fat tissue.

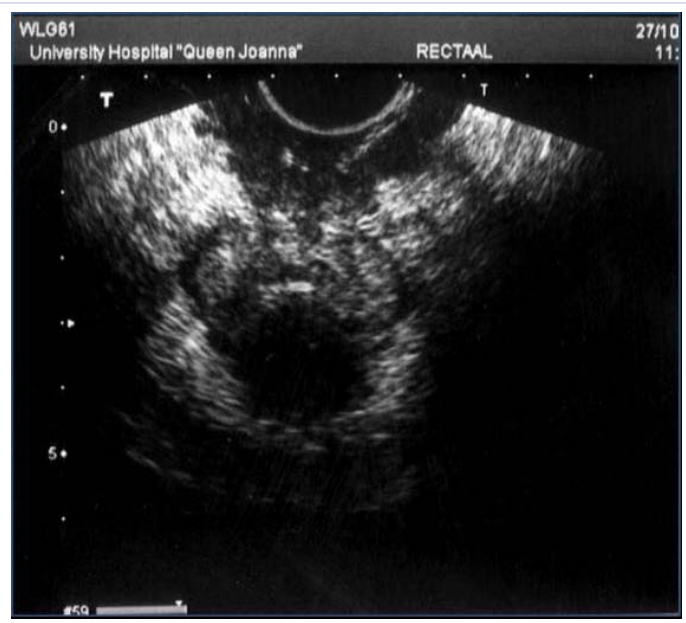

Figure 3: Endosonographic image of the rectal cancer - T4 stage. The tumor infiltrates the prostatic gland. 
from unfilled space in the rectal vault due to insufficient inflation produce strong acoustic shadows and prevent the visualization of the deeper tissues. [1,8]

- In the presence of bulky tumors, the transducer must be placed adjacent to the base of the exophytic lesion and not directly over the surface, so that compression, deformation of the rectal wall and interpretation can be avoided due to deeper infiltration.

- Stenotic lesions do not allow complete examination of the tumor, which can lead to underestimation of the tumor stage. Those are the cases, in which miniprobes passed through standard colonoscopes or endovaginal approach can be used $[12,13]$.

The main disadvantages of ERUS are the dependence from the experience of the operator and also the inability of the method to depict the mesorectal fascia $[10,12,13]$.

\section{Evaluation of $\mathbf{N}$ - stage}

The widespread opinion is that ERUS is less accurate in determining the status of the lymph nodes than the tumor invasion and varies between $60 \%$ and $83 \%[7,8,10]$. The ultrasonographic characteristics that suggest the metastatic nature of lymph nodes are: 1 . Hypoechoic image. 2 . Round shape. 3. Localization close to the main tumor mass (Figure 4).

According to a meta-analysis of more than 2700 patients the sensitivity of the method in determining the N-stage is $73.8 \%$ and the specificity is $75.8 \%$ [14]. Another meta-analysis of publications, including 4118 cases, reports an average accuracy of $75 \%$ [9]. In rectal carcinoma about $50 \%$ of metastatic lymph nodes are smaller than $5 \mathrm{~mm}$; up to $8 \%$ can be smaller than $2 \mathrm{~mm}$ or even smaller $[10,12,15]$. ERUS can miss up to $20 \%$ of these metastatic lymph nodes $[10,16,17]$. An advantage of flexible devices (echoendoscopes) is the ability to reach the iliac zone in search for iliac lymphadenopathy.

Currently ERUS is considered the "gold standard" for preoperative evaluation of rectal cancer [7-14]. It is relatively cheap and available in clinical practice, easy to perform and well tolerated by the patients. The results can be interpreted and discussed "in real time", during the examination.

A lot of publications compare the different imaging modalities for preoperative staging of rectal cancer [18-23]. A systematic review of publications on the use of ERUS and Magnetic Resonance Imaging (MRI) for locoregional staging of rectal cancer (1984-2004) concludes that both techniques are complementary. ERUS is more precise in early lesions (T1 and T2) with overall accuracy of $82 \%$, while MRI, which has an average accuracy of $76 \%$, is more useful for evaluation of T3 and T4 tumors [8]. Both methods show comparable results with the more affordable one being ERUS [18-20].

In our experience, the identification of the lymph nodes close to the tumor during the ERUS is considered to be malignant involvement. At the current time the locoregional staging with

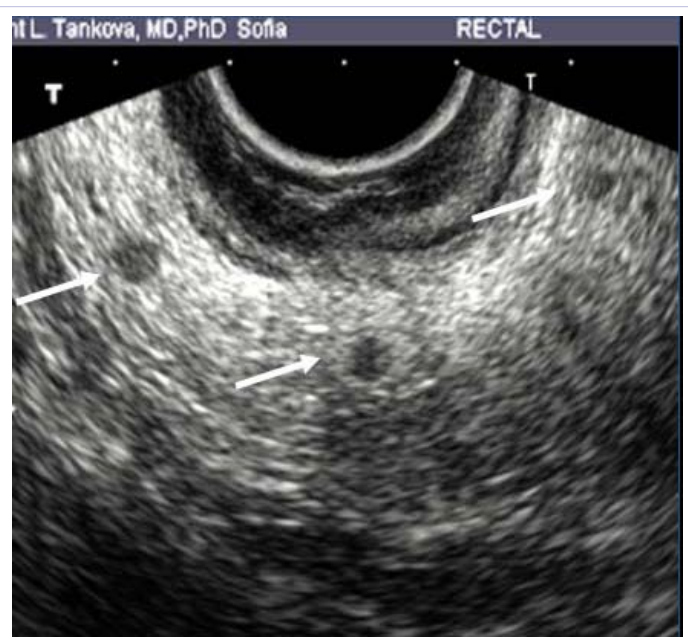

Figure 4: Endosonographic image of the rectal cancer with 3 metastatic lymph nodes (arrows).

ERUS and/or MRI on one hand and examination for systemic metastases with Computed Tomography or Positron Emission Tomography/CT (PET/CT) on the other hand seems to be the best diagnostic regimen for staging of rectal cancer [23-26]. In fact, the choice of the method for local rectal cancer staging depends on the availability of adequate equipment, experts and National consensus.

\section{Ultrasound Methods for Tumor Vascularity Assessment}

The method of evaluating tumor angiogenesis may be useful and informative in both the diagnosis and treatment of rectal cancer. Furthermore, the evaluation of angiogenesis will be clinically important to assess the effectiveness of the antiangiogenic therapy. Some studies have suggested that color Doppler ultrasonography may provide a reliable preoperative quantitation of tumor angiogenesis and prognostic information in cancer patients $[25,26]$.

The color Doppler signals seen within the tumor represent the larger vessels (approximately $100 \mu \mathrm{m}$ or more in diameter), possibly intratumoral arterioles, venules, and arteriole-venule shunts.

Contrast Enhanced Ultrasonography (CEUS) is a wellaccepted and widely available imaging modality in recent years, because it provides a good opportunity for visualization of the microcirculation. CEUS is a promising indirect method of evaluating blood flow within a functional vessel. The secondgeneration contrast agents (e.g. SonoVue) combined with a low-mechanical index ultrasonographic technique based on nonlinear acoustic effects on interactions with microbubbles makes the microbubbles more stable and durable. Meanwhile, the analysis of the Time Intensity Curve (TIC) makes it possible to assess tumor vascularity quantitatively and may have some advantages over the current standard method for quantitative evaluation of angiogenesis-immunofluorescent analysis of 
intratumoral Microvessel Density (MVD), which requires biopsy samples and gives information for the vascularization in a small area of the tumor. However, there is limited experience in using CEUS to assess tumor vascularity in rectal cancer $[27,28]$. The value of TIC parameters in assessing tumor vascularity in rectal cancer remained to be investigated. Maybe in the future contrastenhanced endorectal ultrasound will be applied more widely in the assessment of rectal cancer vascularity and for differentiation of postoperative rectal fibrosis from local recurrence.

At our institute we apply endorectal pulse colour and power Doppler ultrasound as a non-invasive method of preoperative in vivo evaluating the extent of tumor angiogenesis (Figure 5). Our results show that the intensity of the intratumor vascularization, estimated with endorectal Doppler as well as with immunohistochemical methods, correlates to the tumor stage [29]. The value of angiogenesis assessment by endorectal Doppler methods or CEUS perfusion parameters in indicating prognosis of patients with rectal cancer remains to be further investigated.

\section{Alternative Approaches in Ultrasonography of Rectal Tumors}

Transvaginal or endovaginal ultrasound is a valuable addition to endorectal ultrasound in rectal cancer staging [30]. Not much literature exists regarding transvaginal endosonographic assessment of rectal cancer.

This approach gives an information for the status of anal sphincters and is the only ultrasound alternative for examination of women with stenotic tumors, postoperative examination when abdominoperineal extirpation is done or in cases with anterior resection and postoperative stenosis of the rectum. The method is very useful when the data collected with the endorectal examination are insufficient or uncertain.

The layers of the rectal wall seen by transvaginal ultrasound have the same arrangement with alternating hyper and hypoechoic layers, which permits the determination of tumor invasion similar to the endorectal approach $[30,31]$.

The transvaginal ultrasound through clear visualization of the anal sphincter apparatus can be used to assess the integrity of the anal sphincters and the presence of infiltration-valuable information determining the possibility for sphincter-sparing operation (Figure 6).

Transvaginal ultrasonography shows very high specificity and sensitivity, especially in cases of advanced tumors (T3 and T4) and may help in selecting patients suitable for neoadjuvant chemoradiotherapy $[30,31]$. It may be the only ultrasonographic method that may visualize lesions high in the rectum close to the rectosigmoid junction or the stenotic tumors (Figure 7).

Endovaginal ultrasound has an advantage in the assessment of the anal sphincter complex, since in one plane gives information of the sphincters integrity. When endorectal examination is performed with convex, but no radial probe, a rotation of the

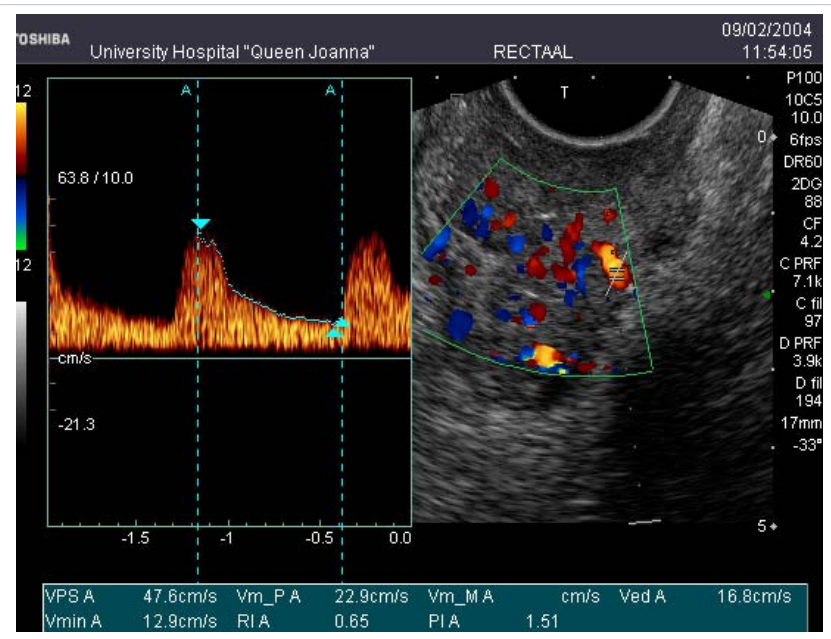

Figure 5: Pulse and Colour Doppler endorectal ultrasonography of the rectal cancer with high vascularization - abundant chaotic vascularity with peak systolic velocity $-47.6 \mathrm{~cm} / \mathrm{sec}$.

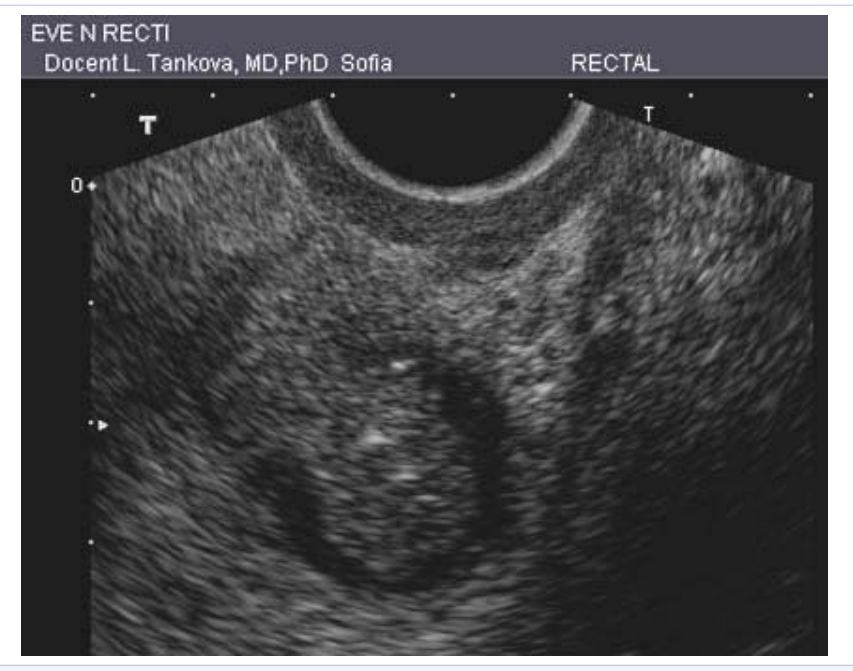

Figure 6: Transvaginal ultrasound - Infiltration of the internal anal sphincter by rectal cancer.

probe is necessary for their complete imaging of anal sphincters.

Transvaginal ultrasound can be done as an addition to every ERUS examination in women with rectal cancer. The method does not prolong the examination substantially, is easily tolerated by most of the patients, usually creates even less discomfort than the rectal approach and gives information about tumor invasion that can improve the accuracy of the combined methods in tumor staging.

In our institute the additional transvaginal ultrasound examination in combination with the ERUS, has become a routine part of the diagnostic and staging process in women with rectal cancer, even in cases with complete and unobstructed endorectal examination as we consider that the combination of both methods gives better results in rectal cancer staging than applying only one method. 

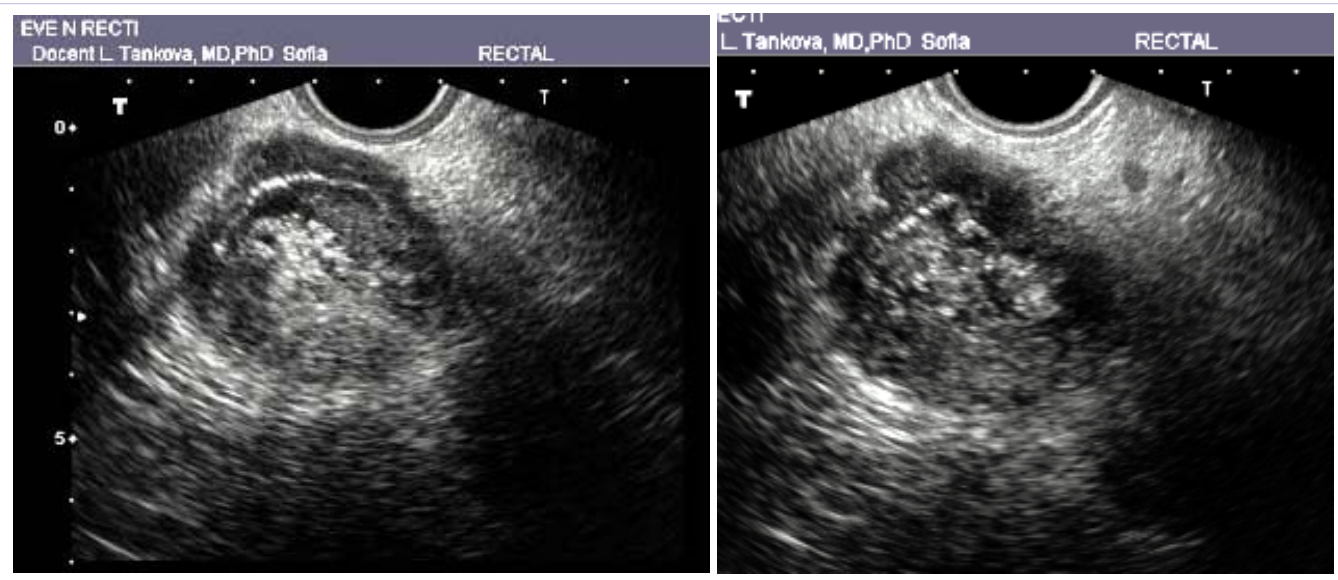

Figure 7: Transvaginal ultrasound - "bulky" rectal tumor with rectal stenosis (arrows); Perirectal lymph node (arrow).

Perineal or perianal ultrasound is very useful in diagnosis of inflammatory perianorectal complications, but it can also successfully be applied in the assessment of the anal canal and the presence of infiltration of the anal sphincters in low rectal or anal neoplasms. The insertion of the rigid endoanal transducer can be very painful, even impossible in cases of anal stenosis. The transcutaneous perineal sonography can be an alternative of the endoanal ultrasound in case of anal stenosis, pain or if the patient refuses the endorectal examination. The investigation is conducted with conventional probes; it is carried out quickly, easily and is well tolerated by the patients. To improve the diagnostic potential of endorectal ultrasound in patients with low rectal or anal tumors we consider perineal ultrasonography as complementary reasonable approach.

\section{Endorectal Ultrasound-Guided Needle Biopsies}

EUS-FNA for rectal cancer has been evaluated for both lymph nodes staging and for detection of extraluminal recurrence [32]. In the preoperative staging of rectal cancer, two studies have evaluated the clinical impact of EUS-FNA, with very similar results [33,34]. Both studies reported that EUS-FNA did not alter significantly the management as compared with EUS alone. It is possible that the lack of clinical impact of EUS-FNA in rectal cancer staging might be related to the close correlation between the $\mathrm{T}$ and $\mathrm{N}$ stages and to the fact that the most perirectal lymph nodes detected at EUS during rectal cancer staging are malignant. In both series, EUS-FNA was significantly more accurate than EUS alone to diagnose malignant recurrence. Ultrasound-guided Fine Needle Aspiration (FNA) biopsy is performed with good success using linear probes, because the path of the needle can be clearly seen $[10,34]$. Some authors use multiplanar probes with transversal scanning for clear visualization of the anatomy and longitudinal for guidance of the needle. With this method the specificity and sensitivity of ERUS for confirming the malignant character of lymph nodes and local recurrence are improved [34]. Contrast enhanced ultrasound techniques compared to conventional ultrasound improves the differential diagnosis of benign lymph nodes from malignant nodes and provide a more accurate selection of nodes to be submitted to fine-needle aspiration biopsy [35].

\section{Three Dimensional ERUS (3D-ERUS)}

Three dimensional ERUS has some benefits in comparison to its 2D counterpart. The method allows better estimation of the volume of the tumor and the relation to nearby anatomical structures. The accuracy for determination of T-stage is $78 \%$ for 3D-ERUS and $69 \%$ for standard ERUS, respectively, for N-stage assessment the accuracy for 3D-ERUS is $65 \%$ and for 2D method is $56 \%$ [36]. Through measuring the change in the volume before and after neoadjuvant therapy 3D-ERUS allows for precise evaluation of the therapeutic response.

\section{Conclusion}

Endorectal ultrasound is the preferred method for rectal cancer staging in early T1 and T2 lesions. In low rectal and anal carcinoma, ERUS and transperineal ultrasound can be used to evaluate the intactness of anal sphincter apparatus. Transvaginal ultrasound can be done as an addition to every ERUS examination in women with rectal cancer. This approach is very useful in cases of rectal stenotic tumors.

\section{References}

1. Hildebrandt U, Feifel G. Preoperative staging of rectal cancer by intrarectal ultrasound. Dis Colon Rectum. 1985; 28(1):42-46.

2. Colaiácovo R, Assef MS, Ganc RL, Carbonari AP, Silva FA, Bin FC, et al. Rectal cancer staging: Correlation between the evaluation with radial echoendoscope and rigid linear probe. Endosc Ultrasound. 2014; 3(3): 161-166. doi: 10.4103/2303-9027.138786

3. Wang Y, Zhou CW, Hao YZ, Li L, Liu SM, Feng XL, et al. Improvement in T-staging of rectal carcinoma: using a novel endorectal ultrasonography technique with sterile coupling gel filling the rectum. Ultrasound Med Biol. 2012; 38(4): 574-579. doi: 10.1016/j. ultrasmedbio.2011.12.020.

4. Kongkam P, Linlawan S, Aniwan S, Lakananurak N, Khemnark $\mathrm{S}$, Sahakitrungruang $\mathrm{C}$, et al. Forward-viewing radial-array echoendoscope for staging of colon cancer beyond the rectum. World J Gastroenterol. 2014; 20(10):2681-2687. doi: 10.3748/wjg.v20. i10.2681. 
5. Benyon J. An evaluation of the role of rectal endosonography in rectal cancer. Ann R Coll Surg Engl. 1989; 71(2): 131-9.

6. Abdool Z, Sultan AH, Thakar R. Ultrasound imaging of the anal sphincter complex: a review. Br J Radiol. 2012; 85 (1015): 865-875. doi: 10.1259/bjr/27314678. Epub 2012 Feb 28.

7. Schaffzin DM, Wong WD. Endorectal ultrasound in the preoperative evaluation of rectal cancer. Clin Colorectal Cancer. 2004; 4(2): 124132

8. Skandarajah AR, Tjandra JJ. Preoperative loco-regional imaging in rectal cancer. ANZ J Surg. 2006; 76(6): 497-504.

9. Harewood GC. Assessment of publication bias in the reporting of EUS performance in staging rectal cancer. Am J Gastroenterol. 2005; 100(4): 808-816.

10. Edelman BR, Weiser MR. Endorectal ultrasound: its role in the diagnosis and treatment of rectal cancer. Clin Colon Rectal Surg. 2008; 21 (3):167-177. doi: 10.1055/s-2008-1080996.

11. Lui ZL, Zhou T, Liang XB, Ma JJ, Zhang GJ. Learning curve of endorectal ultrasonography in preoperative staging of rectal carcinoma. Mol Clin Oncol. 2014; 2(6): 1085-1090.

12. Kav T, Bayraktar Y. How useful is rectal endosonography in the staging of rectal cancer? World J Gastroenterol. 2010; 16(6): 691-697.

13. Samee A, Selvasekar CR. Current trends in staging rectal cancer. World J Gastroenterol. 2011; 17(7): 828-834. doi: 10.3748/wjg.v17.i7.828.

14. Puli SR, Bechtold ML, Reddy JB, Choudhary A, Antillon MR, Brugge WR. How good is endoscopic ultrasound in differentiating various T stages of rectal cancer? Meta-analysis and systematic review. Ann Surg Oncol. 2009; 16(2): 254-65. doi: 10.1245/s10434-008-0231-5.

15. Akasu T, Kondo H, Moriya Y, Sugihara K, Gotoda T, Fujita S, et al. Endorectal ultrasonography and treatment of early stage rectal cancer. World J Surg. 2000; 24(9): 1061-1068.

16. Engelen SM, Beets GL, Beets-Tan RG. Role of preoperative local and distant staging in rectal cancer. Onkologie. 2007; 30(3): 141-145.

17. Giovannini M, Ardizzone S. Anorectal ultrasound for neoplastic and inflammatory lesions. Best Pract Res Clin Gastroenterol. 2006; 20(1): 113-135.

18. Bipat S, Glas AS, Slors FJ, Zwinderman AH, Bossuyt PM, Stoker J. Rectal cancer: local staging and assessment of lymph node involvement with endoluminal US, CT, and MR imaging--a meta-analysis. Radiology. 2004; 232(3): 773-783.

19. Halefoglu AM, Yildirim S, Avlanmis O, Sakiz D, Baykan A. Endorectal ultrasonography versus phased-array magnetic resonance imaging for preoperative staging of rectal cancer. World J Gastroenterol. 2008; 14(22): 3504-3510.

20. Brown G, Davies S, Williams GT, Bourne MW, Newcombe RG, Radcliffe $\mathrm{AG}$, et al. Effectiveness of preoperative staging in rectal cancer: digital rectal examination, endoluminal ultrasound or magnetic resonance imaging? Br J Cancer. 2004; 91(1): 23-29.

21. Heriot AG, Hicks RJ, Drummond EG, Keck J, Mackay J, Chen F, et al. Does positron emission tomography change management in primary rectal cancer? A prospective assessment. Dis Colon Rectum. 2004; 47(4): 451-458.

22.22. Dobos N, Rubesin SE. Radiologicimaging modalities in the diagnosis and management of colorectal cancer. Hematol Oncol Clin North Am. 2002; 16(4): 875-895
23. Bartram C, Brown G. Endorectal ultrasound and magnetic resonance imaging in rectal cancer staging. Gastroenterol Clin North Am. 2002; 31(3): 827-839

24. Kim NK, Kim MJ, Yun SH, Sohn SK, Min JS. Comparative study of transrectal ultrasonography, pelvic computerized tomography, and magnetic resonance imaging in preoperative staging of rectal cancer. Dis Colon Rectum. 1999; 42(6): 770-775.

25. Ogura 0, Takebayashi Y, Sameshima T, Maeda S, Yamada K, Hata $\mathrm{K}$, et al. Preoperative assessment of vascularity by color Doppler ultrasonography in human rectal carcinoma. Dis Colon Rectum. 2001; 44(4): 538-546.

26. Chen CN, Cheng YM, Lin MT, Hsien FJ, Lee PH, Chang KJ. Association of color doppler vascularity index and microvessel density with survival in patients with gastric cancer. Ann Surg. 2002; 235(4): 512-518.

27. Wang Y, Li L, Wang YX, Cui NY, Zou SM, Zhou CW, et al. Time-intensity curve parameters in rectal cancer measured using endorectal ultrasonography with sterile coupling gels filling the rectum: correlations with tumor angiogenesis and clinicopathological features. Biomed Res Int. 2014; 587806. doi: 10.1155/2014/587806.

28.Zhuang H, Yang ZG, Chen HJ, Peng YL, Li L. Time-intensity curve parameters in colorectal tumours measured using double contrastenhanced ultrasound: correlations with tumour angiogenesis. Colorectal Dis. 2012; 14(2): 181-187. doi: 10.1111/j.14631318.2011.02546.x

29. Tankova L, Antonina G, Daniel K, Georgi S, Ivan T. Tumour angiogenesis in rectal cancer-computer-assisted endosonographic and immunohistochemical methods for assessment. London: Intech Open Access Publisher; 2011.

30. Berton F, Gola G, Wilson SR. Perspective on the role of transrectal and transvaginal sonography of tumors of the rectum and anal canal. AJR Am J Roentgenol. 2008; 190(6): 1495-1504. doi: 10.2214/AJR.07.3188

31. Dhamanaskar KP, Thurston W, Wilson SR. Transvaginal Sonography as an Adjunct to Endorectal Sonography in the Staging of Rectal Cancer in Women. AJR Am J Roentgenol. 2006; 187(1): 90-98

32. Tharian B, Tsiopoulos F, George N, Pietro SD, Attili F, Larghi A. Endoscopic ultrasound fine needle aspiration: Technique and applications in clinical practice. World J Gastrointest Endosc. 2012; 4(12): 532-544.

33. Harewood GC, Wiersema MJ, Nelson H, Maccarty RL, Olson JE, Clain JE, et al. A prospective blinded assessment of the impact of preoperative staging on the management of rectal cancer. Gastroenterology. 2002; 123(1): 24-32.

34.Shami VM, Parmar KS, Waxman I. Clinical impact of endoscopic ultrasound and endoscopic ultrasound-guided fine-needle aspiration in the management of rectal carcinoma. Dis Colon Rectum. 2004; 47(1): 59-65.

35. Cui XW, Jenssen C, Saftoiu A, Ignee A, Dietrich CF. New ultrasound techniques for lymph node evaluation. World J Gastroenterol. 2013; 19(30): 4850-4860. doi: 10.3748/wjg.v19.i30.4850.

36. Kim JC, Kim HC, Yu CS, Han KR, Kim JR, Lee KH, et al. Efficacy of 3-dimensional endorectal ultrasonography compared with conventional ultrasonography and computed tomography in preoperative rectal cancer staging. Am J Surg. 2006; 192(1): 89-97. 